\title{
Origem ectópica da artéria subclávia direita e do tronco bicarotídeo no cão
}

\author{
Ectopic origin of the right subclavian artery and the bicarotid trunk in the dog
}

\author{
Paulete de Oliveira Vargas Culau $^{1}$ João César Dias Oliveira ${ }^{2}$ Sueli Hoff Reckziegel $^{3}$ \\ Tânia Lindemann ${ }^{4}$
}

\begin{abstract}
- NOTA -
RESUMO

O suprimento sangüíneo para a cabeça, pescoço, tórax e membros torácicos dos cães provém da artéria aorta através de dois grandes vasos que surgem do arco aórtico, o tronco braquiocefálico e a artéria subclávia esquerda. O presente trabalho descreve a variação de origem da artéria subclávia direita e do tronco bicarotídeo, que foram observados em 8 cães durante 20 anos de dissecções em aulas de anatomia. Nos oito casos examinados, três artérias surgiram do arco aórtico. $O$ primeiro ramo a emergir foi o tronco bicarotídeo, logo após nasceu a artéria subclávia esquerda e imediatamente a seguir, a artéria subclávia direita. O tronco bicarotídeo surgiu do arco aórtico, lateroventralmente à esquerda da traquéia, dirigindose cranialmente e bifurcando-se em nível da primeira costela em artérias carótidas comuns direita e esquerda. Na seqüência, emerge a artéria subclávia esquerda e imediatamente após, surgindo diretamente do arco aórtico, encontra-se a artéria subclávia direita, que se dirige para a margem cranial da primeira costela, estendendo-se dorsocranialmente para cruzar

branches, the brachiocephalic trunk and the left subclavian artery, which arise from the arch of the aorta. The present report described the abnormal origins of the right subclavian and common carotid arteries observed in eigth dogs, during the last twenty years of dissection in anatomy classes. All these eight cases presented three large arteries that left the aortic arch. The first one to arise was a trunk for both common carotid arteries, then a left subclavian artery and finally a right subclavian artery. The bicarotid trunk was originated from the aortic arch at the left side of the trachea and course cranially towards the first rib, where it bifurcated into the right and left common carotid arteries. Following the left subclavian artery arose and after that the right subclavian artery, which arose directly from the aortic arch. This vessel ran towards the first rib and crossed the median plane at the dorsal aspect of the esophagus and the trachea. Both right and left subclavian arteries emitted the usual branches. Although the esophagus was indented by the anomalous vessel, the absence of a cranial dilatation around the sulcus and the good nutritional condition of the animals lead to a conclusion that the swallowing was not significantly affected.
\end{abstract} a linha mediana ao nível da primeira costela, dorsalmente ao esôfago e à traquéia. As artérias subclávia direita e esquerda apresentaram todos os seus ramos sem alterações. Embora o esôfago tenha se apresentado externamente marcado por um sulco, em razão do percurso alterado da artéria subclávia direita, a relativa ausência de dilatação cranial a este e o bom estado nutricional dos animais, nos levaram a supor que o progresso dos alimentos no esôfago não foi afetado significativamente.

Palavras-chave: arco aórtico, artéria subclávia, Canis familiaris.

\section{ABSTRACT}

The blood supply to the head, neck, thorax and thoracic limb come from the aorta artery through its two broad
Key words: aortic arch, subclavian artery, Canis familiaris.

\section{INTRODUÇÃO}

O suprimento sangüíneo para a cabeça, pescoço, tórax e membros torácicos do cão provém da artéria aorta, através de dois grandes vasos que surgem do arco aórtico, o tronco braquiocefálico e a artéria subclávia esquerda (GETTY, 1981; EVANS, 1993). O tronco braquiocefálico, primeiro grande ramo do arco aórtico, passa obliquamente para a direita e cranialmente através da face ventral da traquéia,

${ }^{1}$ Médico Veterinário, Doutor, Professor Adjunto, Departamento de Ciências Morfológicas (DCM), Instituto Ciências Básicas da Saúde (ICBS), Universidade Federal do Rio Grande do Sul (UFRGS), Av Bento Gonçalves 9090, 91540-000, Porto Alegre, RS. Email: anavet@vortex.ufrgs.br.

${ }^{2}$ Médico Veterinário, Mestre, Professor Adjunto, Departamento de Morfologia, Centro de Ciências da Saúde, Universidade Federal Santa Maria, Santa Maria, RS. E-mail: jcd.oliveira@smail.ufsm.br

${ }^{3}$ Médico Veterinário, Mestre, Professor Adjunto, DCM, ICBS, UFRGS.

${ }^{4}$ Médico Veterinário, Doutor, Professor Adjunto do DCM, ICBS, UFRGS. 
apresentando mais ou menos $40 \mathrm{~mm}$ de comprimento e $8 \mathrm{~mm}$ de diâmetro; dele originam-se as artérias carótidas comuns esquerda e direita, com ou sem formação de um tronco bicarotídeo, e a artéria subclávia direita, que surge medial ao primeiro espaço intercostal direito e apresenta mais ou menos $20 \mathrm{~mm}$ de comprimento (EVANS, 1993).

A artéria subclávia esquerda tem sua origem no arco aórtico logo após o tronco braquiocefálico, medial ao terceiro espaço intercostal esquerdo e apresenta mais ou menos $60 \mathrm{~mm}$ de comprimento (EVANS, 1993). Após surgir separadamente da convexidade do arco da aorta, passa cranialmente na face esquerda do esôfago, formando um ligeiro arco (côncavo ventralmente) contornando a primeira costela. Já a artéria subclávia direita correspondente surge opostamente ao primeiro espaço intercostal direito. Conseqüentemente, a artéria subclávia esquerda é relativamente mais longa do que a artéria direita (GETTY, 1981). Os ramos das artérias subclávias direita e esquerda são muito similares. Cada artéria subclávia possui quatro ramos que se originam medialmente à primeira costela e ao primeiro espaço intercostal e que são, em ordem de aparecimento, a artéria vertebral, tronco costocervical, artéria cervical superficial e artéria torácica interna (EVANS \& de LAHUNTA, 2001).

A incidência de variação da origem da artéria subclávia direita em cães é desconhecida. PAIVA (1948) relatou dois casos, VITUMS (1962) três casos em 275 cães, DeKLEER (1970) um caso em 400 cães, TSUKISE et al. (1972) dois casos em 160 cães, GERRITY \& MARTIN (1980) um caso, GRIFFITHS (1989) três casos, e BEZUIDENHOUT (1989) um caso. No entanto, para BEZUIDENHOUT (1992), a origem ectópica da artéria subclávia direita é uma das anomalias vasculares mais comuns em cães. A maioria das variações da origem da artéria subclávia direita foram descobertas acidentalmente em dissecções de aulas de anatomia.

Segundo PAIVA (1948), os dois exemplares observados exibiam a artéria subclávia direita nascendo justaposta crânio-distalmente à artéria subclávia esquerda, tendo, ao cruzar a linha mediana para atingir o membro torácico correspondente, percurso supra-esofágico. Em conseqüência, o primeiro ramo calibroso a ser fornecido pela crossa da aorta era representado pelo tronco das artérias carótidas comuns esquerda e direita, seguindo-se, em ordem, a curta distância e distalmente, as origens das artérias subclávias esquerda e direita. Assim, à particular disposição da artéria subclávia direita se acrescia, nos dois espécimes, a ocorrência do tronco bicarotídeo.
TSUKISE et al. (1972) relataram dois casos de origem ectópica da subclávia direita. No primeiro caso, este vaso se originou diretamente do arco aórtico, entre o tronco comum das artérias carótidas e a artéria subclávia esquerda, e no segundo caso emergiu ao nível da origem da artéria subclávia esquerda, porém dorsalmente a esta. Em ambos os casos, houve formação de um tronco das artérias carótidas comuns que se bifurcava, somente, na artérias carótidas comuns direita e esquerda. Embora apresentando constrição da parede esofágica, o autor não encontrou dilatação deste órgão.

BEZUIDENHOUT (1989) e GRIFFITHS (1989), também relataram que três artérias surgiram do arco aórtico, o primeiro, um tronco para ambas as artérias carótidas (tronco bicarotídeo), que se dirigiu cranialmente ao longo da parede esquerda da traquéia até o nível da alça da subclávia, onde se dividiu em artérias carótidas comuns direita e esquerda. A segunda artéria foi a artéria subclávia esquerda, que se originou da aorta à esquerda do plano mediano e da traquéia, dividindo-se na altura do gânglio estrelado em seus cinco ramos terminais. A terceira artéria a se originar da aorta foi a artéria subclávia direita que passou dorsalmente sobre o esôfago e depois em direção cranioventral até a altura da primeira costela, deixando um sulco profundo na parede esofágica dorsalmente. $\mathrm{O}$ autor, no entanto, não relata nenhum transtorno digestivo.

VITUMS (1962) e GERRITY \& MARTIN (1980) descreveram a formação de dois troncos arteriais surgindo diretamente do arco aórtico, o bicarotídeo e o bissubclávio. Segundo VITUMS (1962), o tronco bicarotídeo apresentava 26 a $31 \mathrm{~mm}$ de comprimento e avançava cranialmente à esquerda da traquéia para bifurcar-se ao nível da primeira costela em artérias carótidas comuns direita e esquerda, seguindo o seu percurso normal em direção à cabeça. O tronco bissubclávio tinha $5 \mathrm{~mm}$ de comprimento, surgindo do arco aórtico na altura da origem normal da artéria subclávia esquerda, dividindo-se em artérias subclávias direita e esquerda. A artéria subclávia esquerda seguiu seu percurso habitual até a altura da primeira costela, ramificando-se conforme padrão habitual. A artéria subclávia direita cruzou dorsalmente ao esôfago dirigindo-se cranioventralmente até a altura da primeira costela, deixando um sulco raso na face dorsal do esôfago. Nenhuma dilatação do esôfago, cranial ao sulco foi observada. A artéria subclávia direita originou os seus ramos usuais continuando-se como artéria axilar.

O presente trabalho objetiva descrever a variação de origem da artéria subclávia direita e do 
tronco bicarotídeo, que foram observados em oito cães durante 20 anos de dissecções em aulas de anatomia.

\section{MATERIAL E MÉTODO}

Dos oito cães utilizados para esta pesquisa, dois foram obtidos durante as dissecções em aulas de anatomia das Faculdades de Veterinária da Universidade Federal do Rio Grande do Sul (UFRGS), e seis da Universidade Federal de Santa Maria (UFSM), durante os últimos 20 anos. Anualmente foram dissecados em torno de 14 cães na UFRGS e 30 cães na UFSM.

Os cães eram animais de rua, sem raça definida, adultos de idades variadas, 5 machos e 3 fêmeas. Os animais foram anestesiados com barbitúrico e após sacrificados com uso de sulfato de magnésio. Imediatamente após foi feita a canulação da artéria carótida comum para a formolização dos espécimes. Estes exemplares foram utilizados nas disciplinas de anatomia dos Cursos de Graduação e Pós-graduação das respectivas Faculdades.

\section{RESULTADOS}

Em todos os oito casos relatados, ao dissecarem-se os vasos que se originavam do arco aórtico, observou-se o primeiro ramo, o tronco bicarotídeo, variando de $21 \mathrm{~mm}$ a $40 \mathrm{~mm}$ de comprimento. Este tronco surgiu lateroventralmente à esquerda da traquéia, dirigindo-se cranialmente e bifurcando-se ao nível da primeira costela em artérias carótidas comuns direita e esquerda.

Logo após a emergência do tronco bicarotídeo no arco aórtico, originou-se a artéria subclávia esquerda e imediatamente, a artéria subclávia direita (Figura 1). Em alguns casos, esta origem era tão próxima que sugeria serem estes vasos oriundos de um tronco comum, quando se examinava o contorno convexo do arco sem deslocar convenientemente a traquéia. No entanto a formação deste tronco comum foi descartada após exame cuidadoso das peças, inclusive com abertura da aorta para observação dos orifícios de origem, totalmente independentes.

A artéria subclávia direita cruzou da esquerda para a direita dorsalmente ao esôfago e então projetou-se cranioventralmente em direção à primeira costela direita. Ao longo deste trajeto, formou um profundo sulco na parede muscular do esôfago (Figura 2).

As artérias subclávias direita e esquerda apresentaram todos os seus ramos sem alterações. $\mathrm{O}$ esôfago, embora consideravelmente marcado pela impressão da artéria subclávia direita, não apresentou dilatação cranialmente e não foi observado nenhum outro tipo de alteração neste órgão ou na traquéia.

\section{DISCUSSÃO}

O arco aórtico, no cão, origina dois grandes vasos, o tronco braquiocefálico e a artéria subclávia

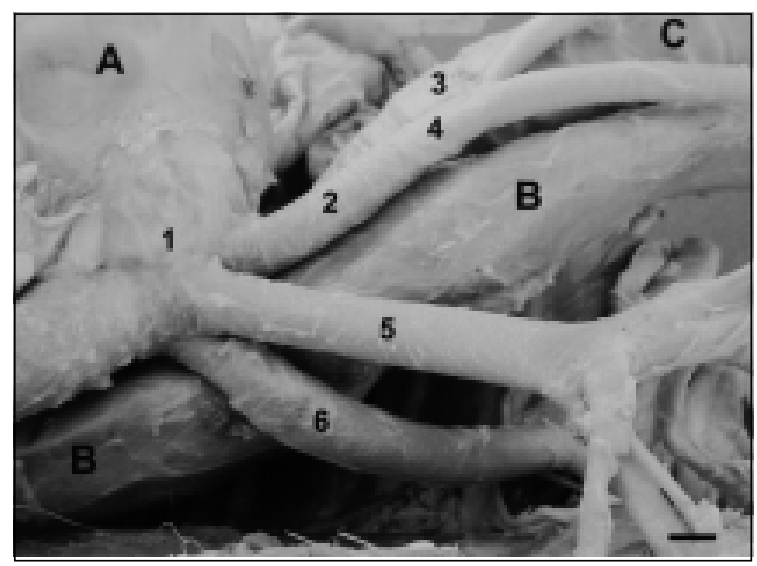

Figura 1 - Vista lateral esquerda do mediastino cranial de cão, mostrando a emergência ectópica da artéria subclávia direita. A - Coração; B - Esôfago; C Traquéia; 1 - Arco aórtico; 2 - Tronco bicarotídeo; 3 - Artéria carótida comum direita; 4 - Artéria carótida comum esquerda; 5 - Artéria subclávia esquerda; 6 - Artéria subclávia direta. (Barra $=0,5 \mathrm{~cm})$.

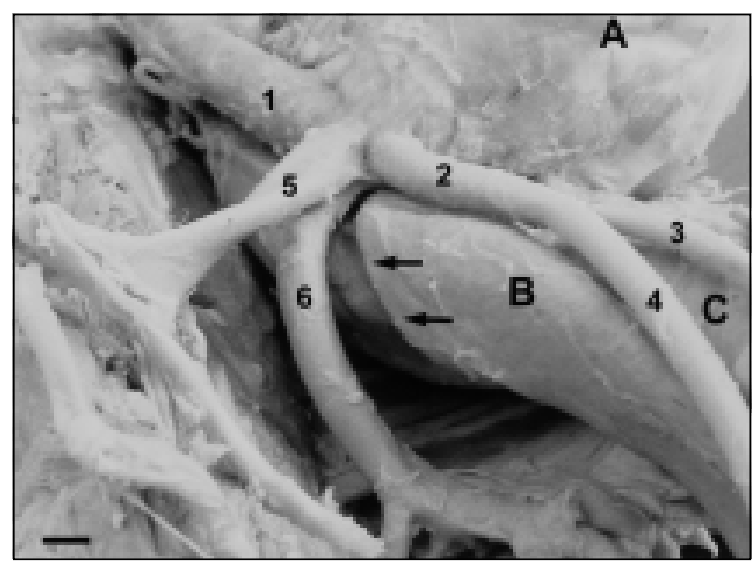

Figura 2 - Vista ventral do mediastino cranial de cão com deslocamento do esôfago. As setas indicam sulco formado na face dorsal do esôfago, em conseqüência do trajeto ectópico da artéria subclávia direta. A Coração; B - Esôfago; C - Traquéia; 1 - Arco aórtico; 2 - Tronco bicarotídeo; 3 - Artéria carótida comum direita; 4 - Artéria carótida comum esquerda; 5 - Artéria subclávia esquerda; 6 - Artéria subclávia direta. $($ Barra $=0,7 \mathrm{~cm})$.

Ciência Rural, v.34, n.5, set-out, 2004. 
esquerda. Do tronco braquiocefálico, emergem as artérias carótidas comuns direita e esquerda, com formação ou não de um tronco bicarotídeo, e a artéria subclávia direita (GETTY, 1981; EVANS, 1993; EVANS \& de LAHUNTA, 2001), padrão esse normalmente observado quando da dissecção do arco aórtico nas salas de estudo anatômico.

A origem direta da artéria subclávia direita e do tronco bicarotídeo da artéria aorta são alterações vasculares que podem resultar de um desenvolvimento anormal do sistema de arcos aórticos. Pesquisando na literatura foram encontrados os relatos de PAIVA (1948) para o qual o provável mecanismo embriogênico que determina essa anomalia ocorre quando o quarto arco aórtico, à direita, atrofia-se inteiramente, enquanto a raiz direita da aorta primitiva dorsal persiste, constituindo a artéria subclávia direita. Já VITUMS (1962) afirma que uma das opções para explicar essa variação seria a persistência da porção distal da raiz aórtica dorsal direita, que na transformação normal é reduzida, e sua união com a sexta artéria intersegmental, constituindo a origem anômala da artéria subclávia direita. Essas explicações embriológicas justificariam a inexpressiva interferência no mecanismo de alimentação e também os achados acidentais nas salas de dissecações, já que os animais se desenvolveram com elas levando a uma adaptação fisiológica.

O comprimento do tronco bicarotídeo, entre $21 \mathrm{~mm}$ e $40 \mathrm{~mm}$, apresentou-se maior que o habitual para esta espécie. Tal fato deveu-se à não formação de um tronco braquiocefálico e também à emergência do primeiro ser diretamente do arco aórtico, essa citação está de acordo com VITUMS (1962), que encontrou o tronco bicarotídeo com $26 \mathrm{~mm}$ a $31 \mathrm{~mm}$ de comprimento e com a de PAIVA (1948), que relata o tronco bicarotídeo com um comprimento de $45 \mathrm{~mm}$.

A impressão inicial de que as artérias subclávias esquerda e direita se originavam por um tronco comum pode ser explicada pela posição de origem da artéria subclávia direita, que surge dorsal à sua homônima esquerda, sendo a primeira totalmente encoberta pela última. VITUMS (1962) e GERRITY \& MARTIN (1980) relataram a formação de um curto tronco comum, que foi por eles denominado de bissubclávio, o que não foi observado em nenhum dos oito casos e tampouco no relato de TSUKISE et al. (1972).

Em relação à emergência ectópica da artéria subclávia direita, esta alteração foi a mais freqüentemente encontrada, em nossas salas de dissecção anatômica, o que está de acordo com DeKLEER (1970) e BEZUIDENHOUT (1992). Tendo em vista que a emergência da artéria subclávia direita ocorreu diretamente do arco aórtico após o surgimento de sua homônima esquerda, o comprimento da artéria direita tornou-se maior que o normal e também mais longo que o da artéria subclávia esquerda do mesmo animal.

Embora o esôfago tenha sido marcado por um sulco, pelo percurso ectópico da artéria subclávia direita, a relativa ausência de dilatação cranial a este sulco e o bom estado nutricional dos animais, sugerem que o trânsito dos alimentos no esôfago não foi significativamente afetado concordando com os relatos de PAIVA(1948), VITUMS (1962), TSUKISE et al. (1972), GERRITY \& MARTIM (1980), BEZUIDENHOUT (1989) e GRIFFITHS (1989).

\section{REFERÊNCIAS BIBLIOGRÁFICAS}

BEZUIDENHOUT, A.J. Anomalous origins of the right subclavian and common carotid arteries in the dog. Journal of South African Veterinary Association, Pretoria, v.60, n.4, p.215-218, 1989 .

BEZUIDENHOUT, A.J. Unusual anomalies of the arteries at the base of the heart in a dog. Journal of South African Veterinary Association, Pretoria, v.63, n.1, p.32-35, 1992.

DeKLEER, V.S. An anomalous origin of the right subclavian artery in the dog. British Veterinary Journal, London, v.127, p.76-82, 1970.

EVANS, H.E. Miller's Anatomy of the dog. 3.ed. Philadelphia : Saunders Company, 1993. 1113p.

EVANS, H.E.; de LAHUNTA, A. Guia para a dissecção do cão. 5.ed. Rio de Janeiro : Guanabara, 2001. 250p.

GERRITY, L.W.; MARTIN, J.E. Anomalous right subclavian artery in a dog. The Southwestern Veterinarian, College Station, v.33, n.1, p.76-77, 1980.

GETTY, R. Anatomia dos animais domésticos. 5.ed. Rio de Janeiro : Interamericana, 1981. 2000p.

GRIFFITHS, D. Three cases of aberrant right subclavian artery in the dog. Acta Veterinaria Scandinavica, Copenhagen, v.30, n.3, p.355-357, 1989.

PAIVA, O.M. Dois casos de A. Subclavia dextra como última colateral do arcus aorticus no cão. Revista da Faculdade de Medicina Veterinária de São Paulo, São Paulo, v.3, n.4, p.203-223, 1948.

TSUKISE, A.; SUGAWA, Y.; OKANO, M. Two anomalous cases of the right subclavian artery arising directly from the aortic arch in dogs. Japanese Journal of Veterinary Science, Tokyo, v.34, p.11-15, 1972.

VITUMS, A. Anomalous origin of the right subclavian and common carotid arteries in the dog. Cornell Veterinarian, Ithaca, v.52, p.5-15, 1962. 